\title{
Reestenose Associada à Fratura de Stent com Liberação de Sirolimus
}

\author{
Alberto Gomes Taques Fonseca', Áurea J. Chaves ${ }^{1}$, José de Ribamar Costa ${ }^{1}$, \\ Rodolfo Staico ${ }^{1}$, Luiz Alberto Mattos ${ }^{1}$, Alexandre Abizaid ${ }^{1}$, Fausto Feres ${ }^{1}$, \\ Amanda G. M. R. Sousa ${ }^{1}$, José Eduardo M. R. Sousa ${ }^{1}$
}

\section{RESUMO}

Os autores descrevem um caso de fratura de stent Cypher $^{\circledR}$. Inicialmente, a fratura foi acompanhada clinicamente (paciente era assintomático, sem evidência clara de isquemia à investigação não-invasiva). Entretanto, durante seguimento tardio, o paciente passou a apresentar sintomas de angina estável, necessitando de nova intervenção percutânea e, posteriormente, implante de novo stent farmacológico.

DESCRITORES: Reestenose coronária. Contenedores. Falha de prótese. Sirolimo.

\section{SUMMARY}

\section{Stent Fracture Associated to Restenosis in Sirolimus Eluting Stent}

The authors describe a case of stent fracture following the use of Cypher $^{\circledR}$. Initially, the fracture was clinically approached (patient was asymptomatic with no clear evidence of ischemia in non-invasive assessment). However, during the long-term follow-up this patient started manifesting stable angina symptoms requiring new percutaneous intervention with additional drug-eluting stent implantation.

DESCRIPTORS: Coronary restenosis. Stents. Prosthesis failure. Sirolimus.

2002, com história de angina aos moderados esforços, estável, após suposto IAM em fevereiro do mesmo ano. O paciente apresentava antecedentes de hipertensão, tabagismo e diabetes. Fazia uso de forma irregular de propanolol $40 \mathrm{mg} / \mathrm{dia}$, captopril $75 \mathrm{mg} / \mathrm{dia}$, hidroclorotiazida $25 \mathrm{mg} /$ dia e ácido acetilsalicílico (AAS) 200 mg/dia. Eletrocardiograma em repouso demonstrava ritmo sinusal e zona eletricamente inativa em parede inferior.

O paciente foi submetido a cineangiocoronariografia, que demonstrou lesão de $50 \%$ no terço médio da artéria descendente anterior (DA), artéria circunflexa (CX) com lesões discretas e lesão suboclusiva em terço médio da artéria coronária direita (CD) (Figura 1A). Ventriculografia esquerda realizada em oblíqua anterior direita (OAD) demonstrou hipocinesia grave no segmento ínfero-médio-basal, volume diastólico final preservado e valva mitral competente. Na época, o paciente foi incluído no estudo Small Vessel Treatment with Cypher ${ }^{\circledR}$ stent (SVELTE).

A intervenção coronária percutânea foi realizada com implante de stent Cypher ${ }^{\circledR} 2,75$ x 33 mm, liberado com 20 atm (Figura 1B), demonstrando resultado final sem lesão residual e com fluxo distal TIMI III (Figura 1C e 1D). A ultra-sonografia intracoronária (USIC) \footnotetext{
Correspondência: Áurea J. Chaves. Av. Dr. Dante Pazzanese, 500 - São Paulo, SP - CEP 04012-180 - Tel.: (11) 5085-6262 - Fax: (11) 5549-7807 • E-mail: achaves@uol.com.br

1 Instituto Dante Pazzanese de Cardiologia - São Paulo, SP. 
realizada em seguida demonstrou boa expansão da prótese, com hastes bem apostas à parede do vaso (Figura 1E). O paciente recebeu alta no dia seguinte, em uso de atenolol $25 \mathrm{mg} / \mathrm{dia}$, enalapril $40 \mathrm{mg} / \mathrm{dia}$, AAS $200 \mathrm{mg} /$ dia, clopidogrel $75 \mathrm{mg} /$ dia, sinvastatina $10 \mathrm{mg} /$ dia e metformina $1.700 \mathrm{mg} / \mathrm{dia}$.

O paciente retornou para controle ambulatorial em 30 dias, apresentando resolução total dos sintomas. Foi orientado a manter as medicações e suspender o clopidogrel após dois meses de uso. Aos seis meses de seguimento, retornou com precordialgia atípica, sem relação com esforço, sendo agendada avaliação angiográfica protocolar com USIC (Figura 2).

No reestudo, realizado oito meses após o procedimento índice, observou-se que a lesão na DA se manti- nha estável (50\%) e que a CX estava sem lesões. O stent, por sua vez, apresentava hiperplasia focal de cerca de 50\% à angiografia coronária quantitativa (ACQ). A USIC demonstrou área luminal mínima de 4,73 mm², associada à imagem de apenas uma haste do stent, confirmando o diagnóstico de fratura do stent. Optouse pela realização, após a alta, de prova farmacológica para avaliar a presença de isquemia. Foi realizada cintilografia de perfusão do miocárdio com Tc-sestamibi, utilizando estresse farmacológico com dipiridamol, que demonstrou ausência de alterações sugestivas de isquemia, com área de hipoperfusão fixa em parede inferior e sem comprometimento da função sistólica global. Na ausência de alterações isquêmicas, optou-se por manter o paciente em tratamento clínico, com controle rigoroso dos fatores de risco.

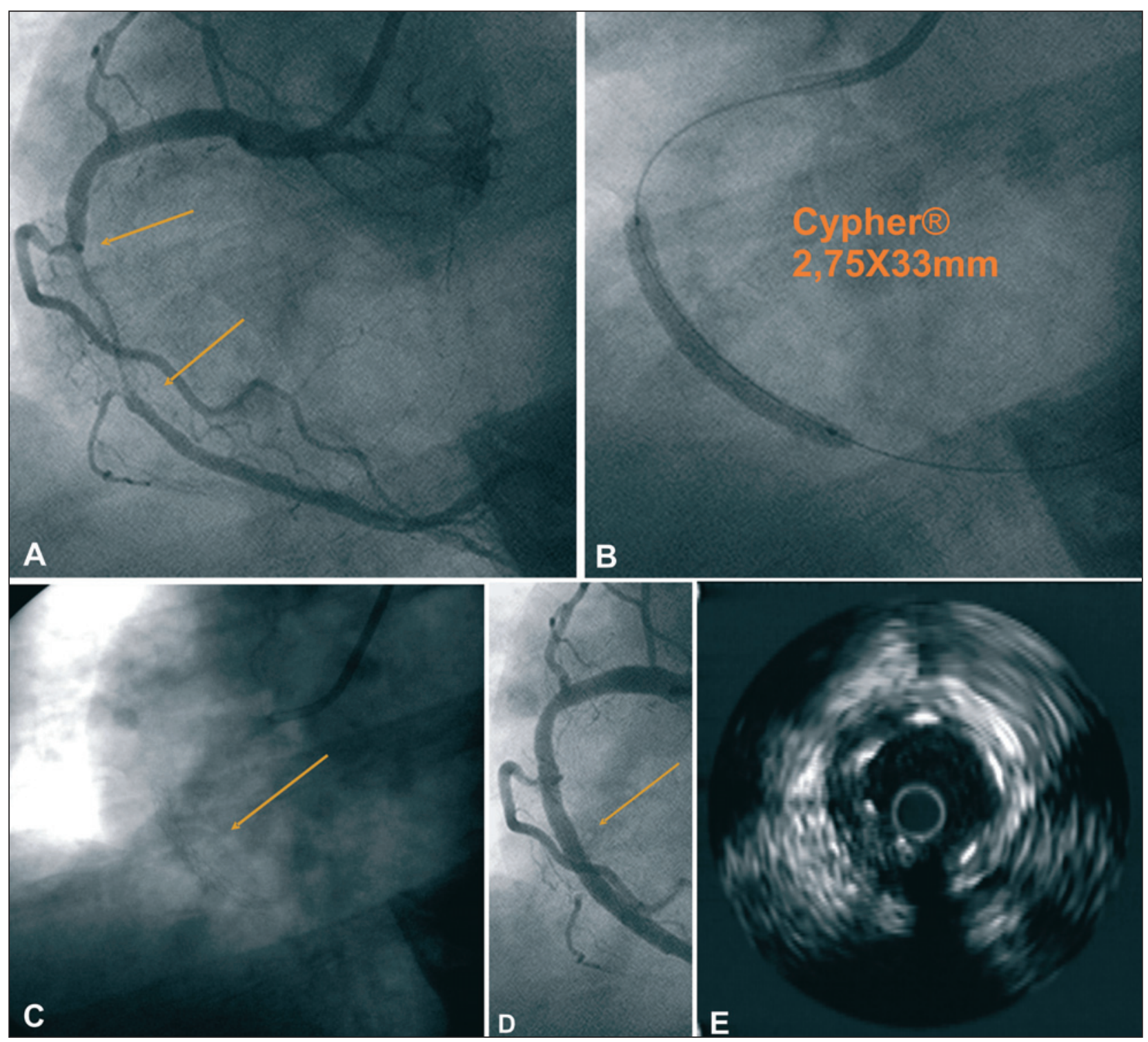

Figura 1 - Angiografia e ultra-sonografia intracoronária durante o procedimento. 
Cerca de dois anos após o implante, o paciente retornou ao ambulatório referindo que, nos 30 dias que precederam a consulta, voltou a apresentar sintomas anginosos semelhantes aos observados na ocasião do implante. Foi submetido a nova avaliação angiográfica (Figura 3), que demonstrou padrão de reestenose focal no terço médio do stent, no local da fratura (A), com lesão quantificada em 66,52\% à ACQ (B) e área luminal mínima de $2,73 \mathrm{~mm}^{2}$ à USIC (C). Diante da reestenose focal associada aos sintomas, e na indisponibilidade de stents com eluição de medicamentos naquele momento, optou-se apenas pela dilatação com cateter-balão Maverick $2^{\circledR} 3,5 \times 20 \mathrm{~mm}$, insuflado com 9 atm (D), alcançando lesão residual inferior a $10 \%(E, F)$, com fluxo distal TIMI III.

O paciente evoluiu sem intercorrências, recebendo alta no dia seguinte. Dez meses após a angioplastia com cateter-balão, foi submetido a novo estudo angiográfico para avaliação dos resultados (Figura 4), que demonstrou recorrência da reestenose, com o
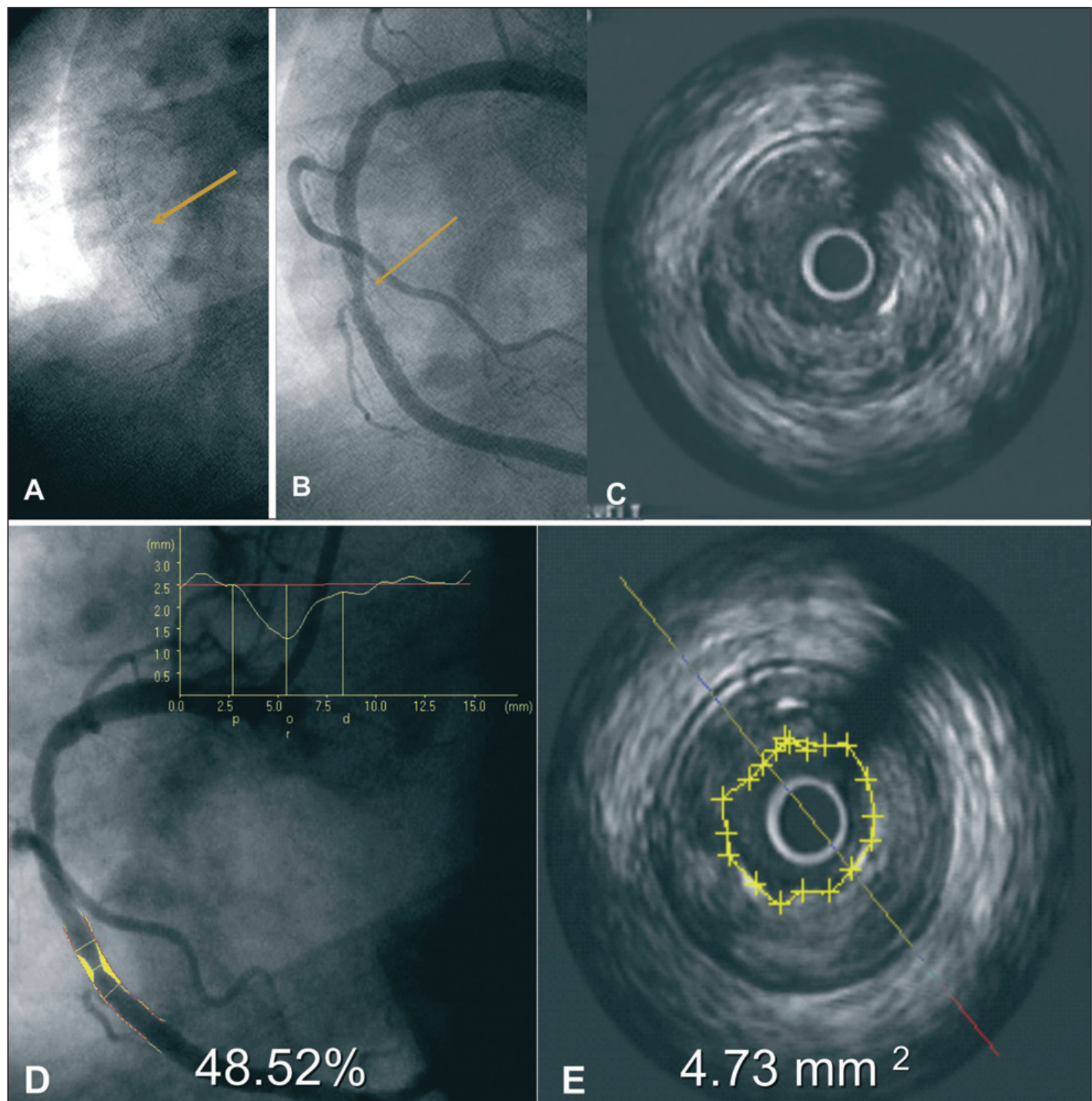

Figura 2 - Imagem demonstrando a separação/fratura das hastes do stent (A). Hiperplasia focal no terço médio do stent (B), acompanhado de apenas uma haste às quatro horas $(C)$ à ultra-sonografia intracoronária (USIC), demonstrando a fratura do stent. A lesão foi quantificada pela angiografia coronária quantitativa (D) e pela USIC (E). 
mesmo padrão focal apresentado anteriormente, sendo realizada nessa ocasião angioplastia coronária com implante de stent Cypher $^{\circledR}$ 3,0 x 18 mm no terço médio da CD. O paciente vem evoluindo assintomático desde o último procedimento. Retornou assintomático à última consulta, em fevereiro de 2007.

\section{DISCUSSÃO}

A primeira descrição de fratura de stent no território coronário data de agosto de 2002, quando Chowdhury e Ramos ${ }^{2}$ descreveram um caso de fratura das hastes de stent implantado doze meses antes, em um enxerto venoso (PS Ao-Mg). Em 2004, Sianos et al. ${ }^{1}$ descreveram os primeiros dois casos de fratura de stent, que ocorreram após implante de stent Cypher ${ }^{\circledR}$ na $C D$, cerca de seis meses após. Atualmente, já foram descritos cerca de 30 casos de fratura de stent Cypher ${ }^{\circledR}$ e apenas um do stent Taxus ${ }^{\circledR 1-11}$; no entanto, sua real prevalência ainda é desconhecida.

Aoki et al. ${ }^{4}$ publicaram estudo realizado no Japão e no Reino Unido, avaliando 280 pacientes tratados com stent Cypher ${ }^{\circledR}$, com avaliação angiográfica e ultra- sonográfica após uma média de 240 dias. Esses autores observaram prevalência de fratura de 2,6\% em locais que trabalhavam como dobradiças durante o movimento do vaso no ciclo cardíaco. Identificaram como preditores de maior risco a extensão do stent e sua localização (enxertos venosos e CD). A presença de fratura no stent aumentou a taxa de reestenose de 12,4\% para $37,5 \%(p=0,07)$ e de novas revascularizações de $11 \%$ para $50 \%(p=0,009)$. Na mesma época, Lee et al. publicaram estudo com 2.728 pacientes submetidos a implante de stents com eluição de medicamentos (3.636 lesões tratadas com stent Cypher $^{\circledR}$ e 1.162 lesões tratadas com stent $\operatorname{Taxus}^{\circledR}$ ), dos quais 530 foram reestudados angiograficamente em decorrência de retorno dos sintomas. Esses autores observaram, nesse estudo, incidência de fratura de hastes de 1,9\% (10 pacientes), com apresentação aguda (angina instável e IAM) em $50 \%{ }^{5}$.

Como já comentado, a fratura das hastes do stent é um evento infreqüente, mas potencialmente grave. Seu espectro clínico pode ir desde o paciente assintomático até os casos de trombose e IAM, passando
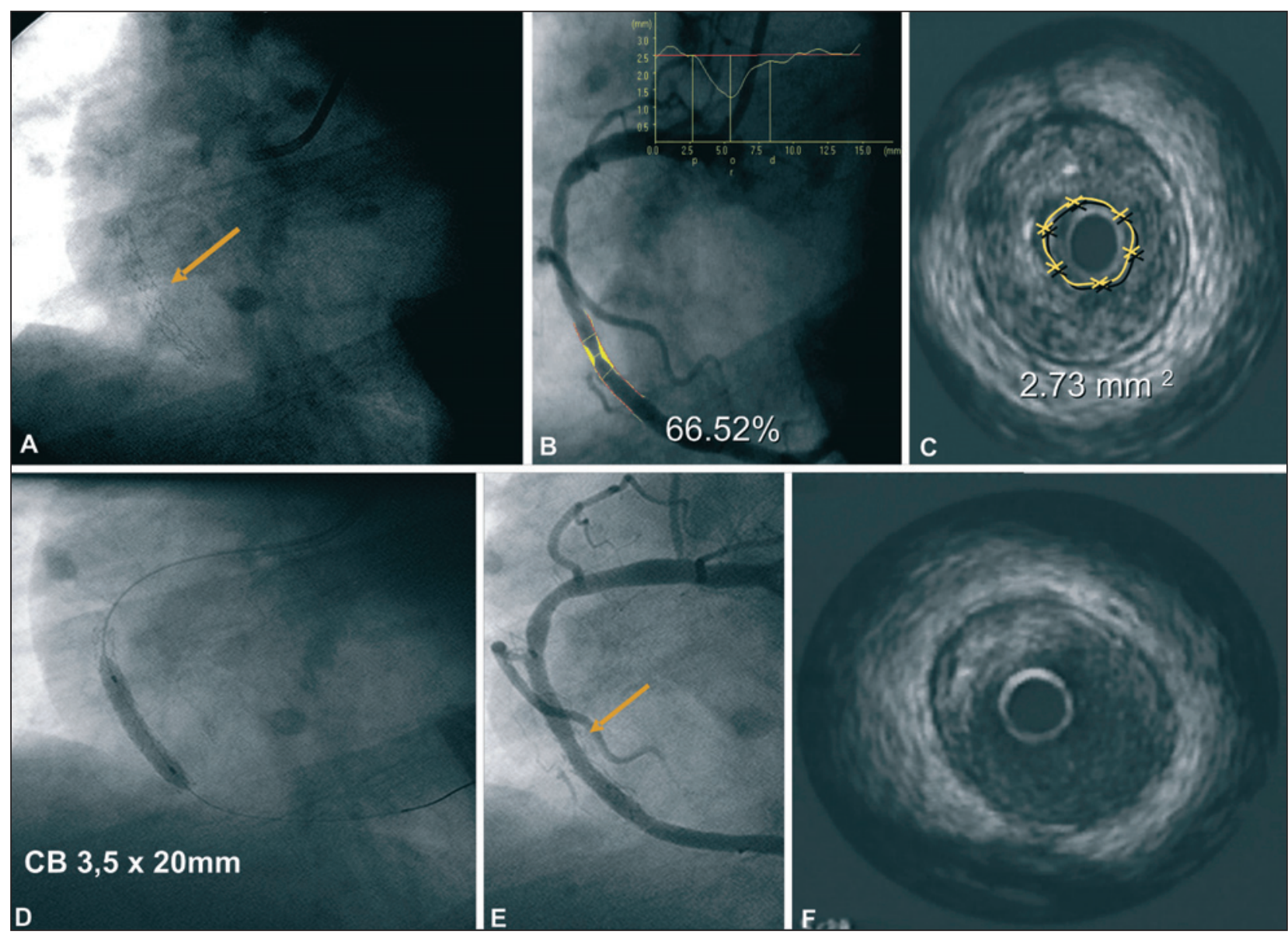

D

Figura 3 - A. Fratura do stent com separação de suas hastes. B. Reestenose focal no terço médio do stent, no local da fratura. C. Área luminal mínima à ultra-sonografia intracoronária. D. Angioplastia com cateter-balão Maverick $2^{\circledR} 3,5 \times 20$ mm. E e F. Resultado final. 
ainda pela reestenose ${ }^{3}$. Dos 30 casos relatados até o momento, 31,8\% apresentaram-se assintomáticos ao reestudo, 31,8\% com angina estável, $13,6 \%$ com angina instável e 22,8\% com IAM.

Tais fraturas estão associadas a áreas de maior rigidez, como os casos de sobreposição de stents (overlap), vasos com mobilidade aumentada, tortuosos e calcificados, e pós-dilatações com balões superdimensionados e altas pressões ${ }^{6}$. O movimento "em dobradiça", conseqüente ao ciclo sístole-diástole, já foi descrito como causa de fratura ${ }^{7}$.

Dos 30 casos relatados até hoje, observa-se que, em $66,7 \%$ deles, havia reestenose associada. Especulase que os mecanismos pelo qual isso ocorra sejam dois: as hastes fraturadas causariam estímulo mecânico à parede do vaso, resultando em inflamação e hiperplasia intimal, e na fratura das hastes a arquitetura do stent fica comprometida, prejudicando a cinética de liberação local do antiproliferativo ${ }^{6}$, gerando o padrão focal de reestenose.

Ainda em relação à reestenose, observa-se que, no caso descrito, ocorreu tardiamente (cerca de 24 meses após o implante do stent). Tal fato talvez seja explicado pelo padrão temporal da reestenose após implante de stent farmacológico. Nesses pacientes, contrariamente aos stents não-farmacológicos, em que o pico na formação da hiperplasia neointimal ocorre aos seis meses e a partir daí tende a se estabilizar ou até mesmo a diminuir, a hiperplasia pode aumentar ao longo do tempo. Esse fato foi observado em estudo conduzido com pacientes tratados com stents com eluição de sirolimo, submetidos a USIC seriadas, em que o porcentual de hiperplasia foi de 2,2\% em um ano, de 3,3\% aos dois anos, e de $5,7 \%$ aos quatro anos ${ }^{8-10}$.

Chama a atenção a virtual ausência de relatos de fratura de hastes com os stents não-farmacológicos. Na verdade, esses stents também estavam e estão sujeitos a fraturas. No entanto, o desalinhamento de suas hastes acabava sendo mascarado pela ampla reendotelização e hiperplasia intimal presente em seu interior ${ }^{11}$. Como nos stents farmacológicos há supressão agressiva na hiperplasia neointimal, e tal supressão está relacionada diretamente à liberação local do fármaco, as hastes fraturadas com pouca hiperplasia ao redor do stent tornam-se mais visíveis ${ }^{12}$.

Corroborando o citado anteriormente, o stent $\mathrm{Bx}$ Velocity ${ }^{\circledR}$ (Cordis) foi e ainda é muito utilizado em centros que não dispõem de stents farmacológicos para uso rotineiro, não havendo nenhum relato de fratura em suas hastes na literatura. Considerando os 30 casos
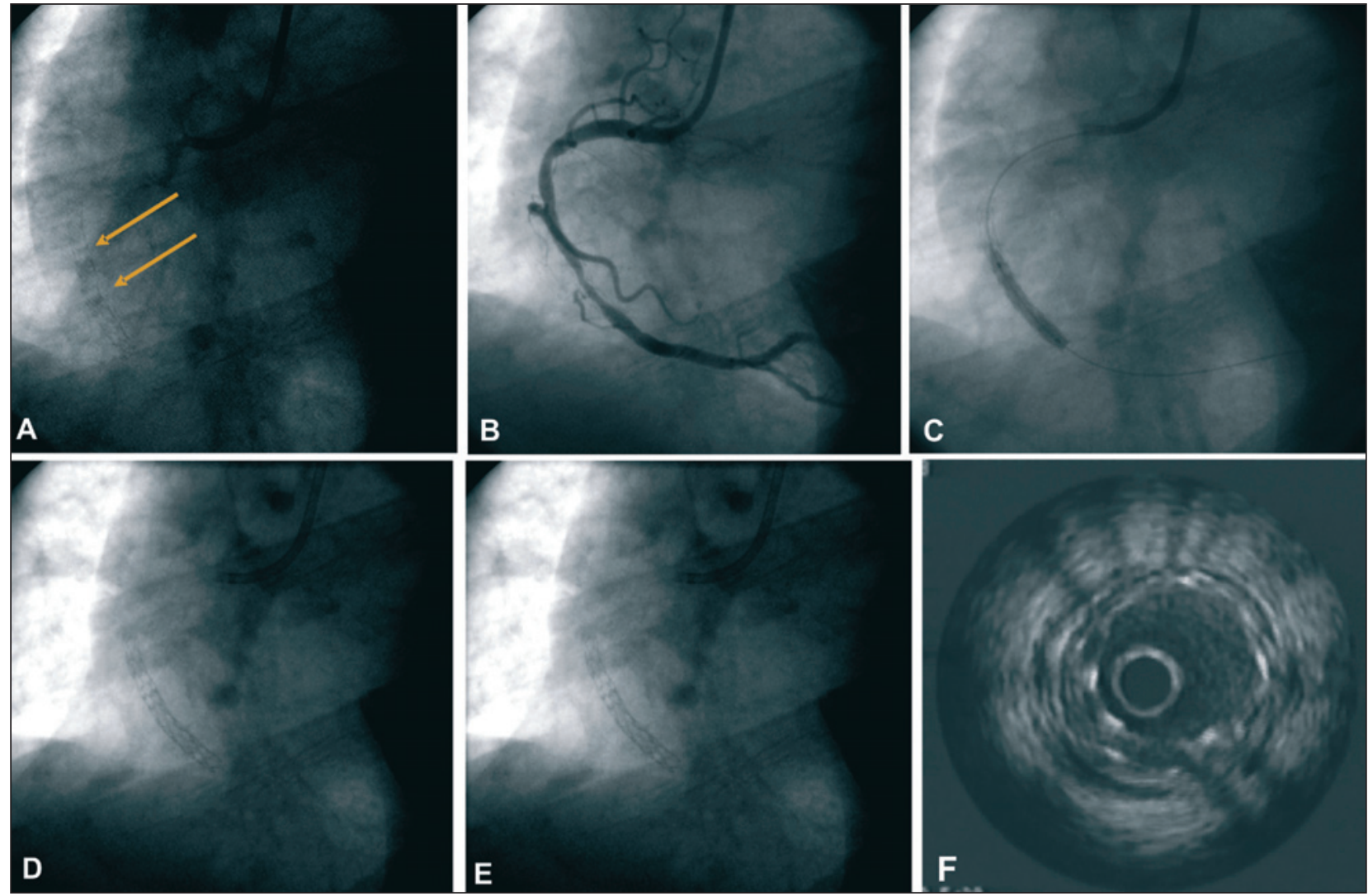

Figura 4 - A. Presença de stent com separação de suas hastes. B. Recorrência da reestenose. C. Implante do stent Cypher ${ }^{\circledR}$. D, E e F. Resultado final. 
já relatados previamente com o stent Cypher $^{\circledR}$, alguns autores especulam que o polímero poderia estar implicado na gênese do problema ${ }^{11}$.

Ainda em relação à diferença do número de casos de fratura publicados $\left(\right.$ Cypher $^{\circledR}$ vs. Taxus $\left.{ }^{\circledR}\right)$, observamos que existe apenas um caso de fratura relatado com o stent Taxus ${ }^{\circledR 13}$. Supõe-se que tal diferença poderia estar relacionada ao desenho do stent, visto ter o Cypher ${ }^{\circledR}$ desenho com células fechadas, com ângulos entre as hastes muito menores, mais suscetível a fraturas em relação ao Taxus $^{\circledR}$, cujo desenho apresenta células mais abertas ${ }^{12}$.

Em relação ao diagnóstico, a separação das hastes tem sido avaliada em alguns casos por meio de fluoroscopia e de USIC, embora a tomografia computadorizada com múltiplos detectores também tenha demonstrado resultados positivos ${ }^{14}$. Em casos de maior risco (stents longos, implantados com altas pressões, CD), Wilczynska et al. ${ }^{5}$ têm sugerido submeter esses pacientes a fluoroscopia como rotina no seguimento.

\section{REFERÊNCIAS BIBLIOGRÁFICAS}

1. Sianos G, Hofma S, Ligthart JM, Saia F, Hoye A, Lemos PA, et al. Stent fracture and restenosis in the drug-eluting stent era. Catheter Cardiovasc Interv. 2004;61(1):111-6.

2. Chowdhury PS, Ramos RG. Images in clinical medicine. Coronary-stent fracture. N Engl J Med. 2002;347(8):581.

3. Makaryus AN, Lefkowitz L, Lee AD. Coronary artery stent fracture. Int J Cardiovasc Imaging. 2007;23(3):305-9.

4. Aoki J, Nakazawa G, Tanabe K, Hoye A, Yamamoto H, Nakayama $\mathrm{T}$, et al. Incidence and clinical impact of coronary stent fracture after sirolimus-eluting stent implantation. Catheter Cardiovasc Interv. 2007;69(3):380-6.
5. Wilczynska J, Rdzanek A, Kochman J, Horszczaruk GJ, Pietrasik A, Opolski G, et al. Sirolimus eluting stent fracture following angioplasty of diffuse in-stent restenosis in the right coronary artery. Int J Cardiol. 2007;118(1):126-7.

6. Jin X, Zhang $S$, Xie $H$, Wang $C$, Fan Z, Zeng $Y$, et al. Strut fracture of DES: an increasing problem? Int J Cardiol. 2007; 118(2):e54-6.

7. Yagi S, Kimura T, Hayashi I, Nishiuchi T. Acute coronary syndrome due to hinge movement of a bare-metal stent. Int J Cardiol. 2007; [Epub ahead of print]

8. Sousa JE, Costa MA, Abizaid AC, Rensing BJ, Abizaid AS, Tanajura LF, et al. Sustained suppression of neointimal proliferation by sirolimus-eluting stents: one-year angiographic and intravascular ultrasound follow-up. Circulation. 2001;104 (17):2007-11.

9. Sousa JE, Costa MA, Sousa AG, Abizaid AC, Seixas AC, Abizaid AS, et al. Two-year angiographic and intravascular ultrasound follow-up after implantation of sirolimus-eluting stents in human coronary arteries. Circulation. 2003;107(3): 381-3.

10. Sousa JE, Costa MA, Abizaid A, Feres F, Seixas AC, Tanajura LF, et al. Four-year angiographic and intravascular ultrasound follow-up of patients treated with sirolimus-eluting stents. Circulation. 2005;111(18):2326-9.

11. Min PK, Yoon YW, Moon Kwon H. Delayed strut fracture of sirolimus-eluting stent: a significant problem or an occasional observation? Int J Cardiol. 2006;106(3):404-6.

12. Kim EJ, Rha SW, Wani SP, Suh SY, Choi CU, Kim JW, et al. Coronary stent fracture and restenosis in the drug-eluting stent era: do we have clues of management? Int J Cardiol. 2007;120(3):417-9.

13. Hamilos MI, Papafaklis MI, Ligthart JM, Serruys PW, Sianos G. Stent fracture and restenosis of a paclitaxel-eluting stent. Hellenic J Cardiol. 2005;46(6):439-42.

14. Zaizen H, Tamura A, Miyamoto K, Nakaishi T, Kadota J. Complete fracture of sirolimus-eluting stent detected by multislice computed tomography. Int J Cardiol. 2007;118(1):120-1. 